\title{
Detection of label-free cancer biomarkers using nickel nanoislands and quartz crystal microbalance
}

This article was published in the following Dove Press journal:

International Journal of Nanomedicine

4 September 2010

Number of times this article has been viewed

\section{Adrián Martínez-Rivas ${ }^{1,2}$ \\ Patrick Chinestra ${ }^{3,4}$ \\ Gilles Favre ${ }^{3,4}$ \\ Sébastien Pinaud' \\ Childérick Séverac ${ }^{1,2}$ \\ Jean-Charles Faye ${ }^{3,4}$ \\ Christophe Vieu ${ }^{1,2}$}

'LAAS-CNRS; Université de Toulouse, Toulouse, France; ${ }^{2}$ Université de Toulouse, UPS, INSA, INP, ISAE; LAAS; Toulouse, France; ${ }^{3}$ INSERM U563, Université de Toulouse, CPTP, "Signalisation Cellulaire, GTPase Rho et cancers", Toulouse, France; ${ }^{4}$ Institut Claudius Regaud, Biology Department, Toulouse, France
Correspondence: Adrian Martínez-Rivas CNMN; Instituto Politécnico Nacional, Luis Enrique Erro s/n, Col. Zacatenco D.F., Mexico Email nanobiomex@hotmail.com

\begin{abstract}
We present a technique for the label-free detection and recognition of cancer biomarkers using metal nanoislands intended to be integrated in a novel type of nanobiosensor. His-tagged (scFv)-F7N1N2 is the antibody fragment which is directly immobilized, by coordinative bonds, onto $\sim 5 \mathrm{~nm}$ nickel islands, then deposited on the surface of a quartz crystal of a quartz crystal microbalance (QCM) to validate the technique. Biomarker GTPase RhoA was investigated because it has been found to be overexpressed in various tumors and because we have recently isolated and characterized a new conformational scFv which selectively recognizes the active form of RhoA. We implemented a surface chemistry involving an antibiofouling coating of polyethylene glycol silane (PEG-silane) $(<2 \mathrm{~nm}$ thick) and Ni nanoislands to reach a label-free detection of the active antigen conformation of RhoA, at various concentrations. The methodology proposed here proves the viability of the concept by using Ni nanoislands as an anchoring surface layer enabling the detection of a specific conformation of a protein, identified as a potential cancer biomarker. Hence, this novel methodology can be transferred to a nanobiosensor to detect, at lower time consumption and with high sensitivity, specific biomolecules.
\end{abstract}

Keywords: nickel nanoislands, cancer biomarkers, quartz crystal microbalance, PEG-silane, RhoA protein, nanobiosensor

Nanomedicine is emerging as one of the most exciting and promising application of nanobiotechnologies, increasing diagnostic possibilities and enabling the implementation of a personalized medicine. ${ }^{1,2}$ Nanobiotechnologies are expected to give rise to applications in vivo and in vitro that will both increase reliability of early diagnosis and beat the disease while it is at its weakest development stage. ${ }^{3}$

Nanoislands are ultrafine particles of a few to several hundred atoms, exhibiting a huge surface to volume ratio, explaining their potential reactivity with the environment and so their usefulness for highly sensitive bimolecular detection. Moreover, when very small nanoislands are used, the chances for protein denaturation can be minimized. ${ }^{4}$ For in vivo biomedical applications, the use of nanoislands presenting superparamagnetic behavior at room temperature is preferred, ${ }^{5}$ such as nanoislands of nickel, cobalt, or iron. Nanoislands of ferromagnetic metals have been intensively studied, due to their interesting physical properties, and used as catalysts for highdensity magnetic recording media or for single electron devices. ${ }^{6,7}$ However, although these have been utilized in nanoelectronics or in vivo nano-objects applications, they have never been used for in vitro biodetection of cancer biomarkers. Ni nanoislands are especially interesting due to the possible stable attachment of proteins on their 
surfaces. The purification method developed by Porath et $\mathrm{al}^{8}$ is currently used in the microbiological protocols; it consists of binding proteins onto immobilized metal ion affinity chromatography (IMAC). The purification is possible because of the affinity the proteins with exposed amino acid cysteine or histidine side chains have, for metals such as $\mathrm{Ni}^{2+}, \mathrm{Co}^{2+}, \mathrm{Zn}^{2+}, \mathrm{Cu}^{2+}$, and $\mathrm{Mn}^{2+}$, through coordinative bonds. The nitrogen in the imidazole core histidine of $6 \mathrm{His}$-tagged proteins binds to $\mathrm{Ni}^{2+}$ cations, and the dissociation constant has been reported to be around $10^{-13} \mathrm{M}$ in the case of His Ni-NTA at $\mathrm{pH} 8 .^{9}$ In this work, we investigate this principle by using a very thin layer of Ni exhibiting nanoislands, coupled with a surface chemistry for attaching His-tagged antibody fragments for the ligand-binding assays.

A biomarker is described as an entity which can reveal through its concentration measurement either a normal biologic (pathogenic) process or a pharmacologic response to a therapeutic intervention. Cancer biomarkers such as prostate-specific antigens (PSA) refer to molecules in which the level of expression is changed in the tumor or in the blood, urine, or other body fluid of cancer patients. ${ }^{10}$ Beyond early detection, the information provided by biomarkers helps in determining the status of the disease, hence improving control and prevention of cancer. ${ }^{11}$ Rho GTPases are molecular switches which control a wide variety of signal transduction pathways in all eukaryotic cells. The roles of Rho GTPases in cells have been identified ${ }^{12}$ as controlling the dynamics of microtubules and actin cytoskeleton and influencing cell polarity. Their activity is regulated by GDP/GTP cycling, functioning in many cellular pathways, controlling proliferation. Hence, they are involved in almost every stage of tumorigenesis. ${ }^{13}$ The expression of some Rho family members such as Rho A or Rho B has been found to be increased or decreased in some human cancers. ${ }^{14}$ These reasons are why we were interested in Rho GTpases as potential cancer biomarkers. However, only the active GTP-bound Rho forms are able to activate the signaling pathway involved in tumorigenesis. This raises the issue of being able to detect specifically the active form of this biomarker. The challenge, related to the ability to sense the conformation of a protein with a nanobiosensor forms the core of this work. For achieving this purpose, we have recently identified a conformational-specific single chain fragment variable ( $\mathrm{scFv}$ ), using phage display techniques, that recognizes the active GTP-bound forms of Rho A, B, and $\mathrm{C}$ and is able to discriminate these from the inactive GDP-bound form. ${ }^{15}$
For validating the biomolecular interaction and the specific recognition, we used a quartz crystal microbalance (QCM). This technology permits the determination of an additional mass per unit area by measuring the frequency shift of the mechanical resonance of a quartz crystal. That is, when there is adsorption of (bio)molecules onto the surface of a piezoelectric quartz, decrements in its resonance frequency are registered this relationship being governed by the Sauerbrey equation. Hence, this technique enables the quantification of deposited masses at the surface of the quartz crystal and the characterization of the viscoelastic properties of thin adsorbed films. QCM technology is particularly well adapted for the development and validation of biofunctionalized surfaces or current protein chips applications. ${ }^{16}$ The resolution in frequency has been reported to be in the range of $0.2 \mathrm{~Hz}$ (in liquid), corresponding to a mass resolution of few $\mathrm{ng} / \mathrm{cm}^{2} .^{17}$

Thanks to an innovative surface chemistry based on PEGSilane and Ni nanoislands deposited onto the thin layer of an $\mathrm{SiO}_{2}$-quartz crystal, we were able to selectively detect an active form of this potential biomarker using QCM technology. The full chemical and biological protocol validated by this experiment can be reproduced on any integrated nanobiosensor such as micro/nanocantilevers, ${ }^{18,19}$ resonant micromembranes, ${ }^{20}$ or interdigitated nanoelectrode devices. ${ }^{21,22}$

Specifically, this technique is being transferred to a novel type of ultrasensitive nanobiosensor (not presented here) based on multiple tunnel junctions (MTJ). These devices fabricated on silicon technology (e-beam lithography and lift-off) use nickel nanoislands, as used here, deposited between interdigitated nanoelectrodes and on a silicon oxidized substrate, which allow high sensitivities to be demonstrated. This is why we used $\mathrm{SiO}_{2}$ quartz surfaces decorated with very small Ni nanoislands in this study. Hence, the present publication validates the adsorption of the probe molecules (conformation specific antibody anti-RhoA) on the Ni nanoislands through His linkage, the efficiency of the antibiofouling layer around the islands, and the capability of our antibodies to detect specific conformational modifications of RhoA cancer biomarkers in a label-free manner. Finally, it is important to note that we used the QCM technology to validate the surface chemistry on a surface, as similar as possible as the surface of our MTJ devices that we are constructing, to demonstrate and report, in future work, high sensitivities.

\section{Material and methods}

The measurements were performed using a QCM (Q-sense $\mathrm{E} 4$, Sweden). The quartz had $50 \mathrm{~nm} \mathrm{SiO}_{2}$ on the surface 
(Q-sense, Sweden) and was then covered with $1 \mu \mathrm{m}$ silicon oxide $\left(\mathrm{SiO}_{2}\right)$ using plasma-enhanced chemical vapor deposition (PECVD), in our clean room facility. For control experiments, we used a fluorescence microscope (Olympus IX70).

\section{Antibiofouling coating}

For many application purposes, related to micro and nanobiosensors, silicon oxide surfaces are used due to their compatibility with silicon technology. We have thus optimized a chemical protocol on this type of surface. For this reason we have employed quartz crystals covered with a silicon oxide thin layer. During the experiments, we immediately noticed that the biomolecules of interest for our work (antibody fragments and proteins) exhibited some unwanted interactions with those $\mathrm{SiO}_{2}$ surfaces. We therefore tested several passivation methods to avoid these non-specific adsorptions. We decided to use 2 [methoxy(polyethyleneoxy) propyl]trimethoxy silane (Gelest), (Mw 460-590), a silane conjugated polyethylene-glycol chain (PEG-silane) because of its biocompatibility, and its covalent binding to $\mathrm{SiO}_{2}$. This kind of PEG has already been used in nanostructured PEGfilms preparations for silicon-based BioMEMS. ${ }^{23}$ Briefly, PEG-silane molecules and concentrated hydrogen chloride $(\mathrm{HCl})$ were mixed in toluene in a clean glass beaker with $100 \mu \mathrm{L}$ PEG-silane, $40 \mu \mathrm{L} \mathrm{HCl}$, and $50 \mathrm{~mL}$ of toluene. The beaker was then placed in a sonicator for 1 minute and the contents were then transferred to another clean glass beaker. A $1.5 \mathrm{~cm}$-diameter piece of quartz, covered with $1 \mu \mathrm{m}$ $\mathrm{SiO}_{2}$-thick (PECVD-deposited layer) was immersed for 2 hours and washed sequentially with toluene, ethanol, and finally deionized water (each step taking 2 minutes). This protocol generates an ultrathin PEG layer on the surface with a thickness of between $1 \mathrm{~nm}$ and $2 \mathrm{~nm} .{ }^{24}$ The quartz surface after this functionalization process is schematized in Figure 1.

\section{Nickel nanoislands deposition}

Different techniques exist for depositing nanoislands, including sputtering or vacuum evaporation. The sputtering method was disregarded because we did not want the deposition process to damage the PEG coating, which

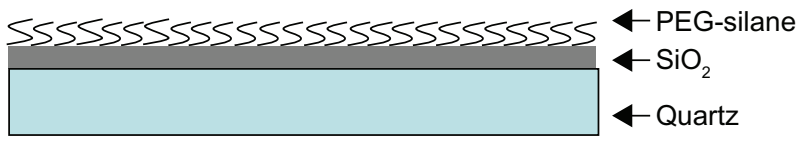

Figure I Schematic representation of a quartz functionalized with an anti-fouling PEG-silane coating. the sputtering method would have caused due to its ionic bombardment energy.

We used a Veeco 770 thermal evaporator to evaporate a $1 \mathrm{~nm}$ thick Ni film onto the PEG-silane surfaces, at a pressure of $\sim 10^{-6}$ mbars at room temperature (RT). For this very low thickness, the Ni film is not continuous and spontaneously adopts a $3 \mathrm{D}$ growth mechanism leading to the formation of nanoscale metallic islands. This morphology was confirmed by high-resolution scanning electron microscopy (SEM) (Hitachi S-4800). A typical image of the deposited film is shown in Figure 2.

The Ni nano islands appear as tiny bright spots and a statistical analysis of the observed nanoislands indicated they had an average diameter of $5 \mathrm{~nm}$. The characterization of the thin Ni film was confirmed by atomic force microscopy (AFM). Images were obtained in air and in contact mode, of a JPK system (NanoWizard ${ }^{\circledR}$ II; JPK Instruments, Berlin, Germany) with a force evaluated to $200 \mathrm{pN}$. A typical image is shown in Figure 3.

Using AFM imagery the nanoislands appear larger compared to SEM due to tip convolution effects which tend to render hard material objects larger than they are. However, the height analysis provided by AFM indicates an average vertical dimension (close to $5 \mathrm{~nm}$ ) in very good agreement with the lateral dimension extracted from high magnification SEM. Both images (SEM and AFM) indicate that there are nanometric gaps between the Ni nanoislands and that their coalescence was very limited. In our process, these Ni nanoislands attach to the antibody fragments through Ni-histidine binding. We expect this kind of morphology to be vaulable for minimizing the possible denaturation of adsorbed biomolecules on the surface. The final surface of the quartz crystals used for the QCM experiments is depicted in Figure 4.

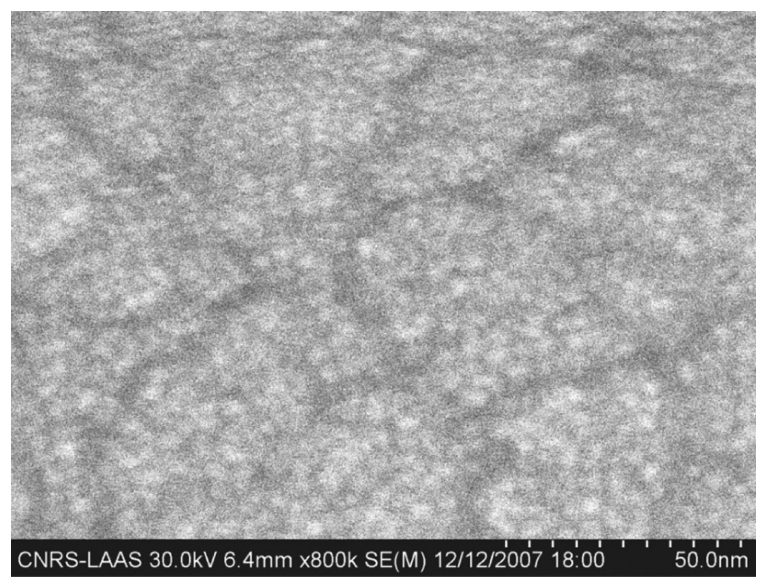

Figure 2 High-resolution SEM image of a granular Ni film (I nm nominal thickness) deposited by thermal evaporation onto $\mathrm{a} \mathrm{SiO}_{2}$ surface. 


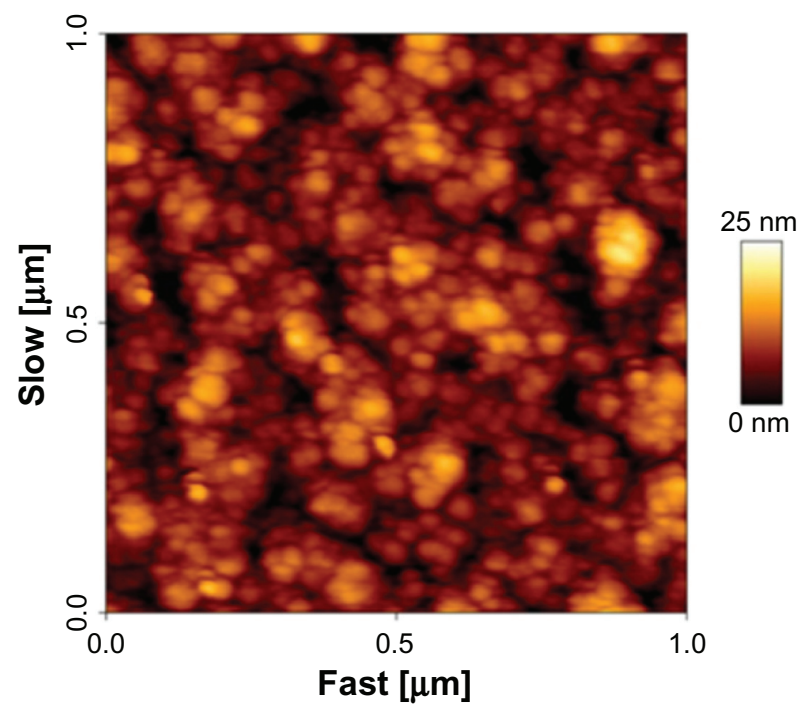

Figure 3 AFM image of a granular Ni film ( $\mathrm{nm}$ nominal thickness) deposited by thermal evaporation onto a $\mathrm{SiO}_{2}$ surface.

\section{Biomolecule purification}

The production and purification of soluble single-chain antibodies was performed as follows: TG1 Escherichia coli strain harboring the pHEN F7N1N2 plasmid was grown in $100 \mathrm{~mL}$ of $\mathrm{LB}$ medium containing ampicillin $(100 \mu \mathrm{g} / \mathrm{mL})$ and $2 \%$ of glucose at $37^{\circ} \mathrm{C}$. At a proper concentration $\left(\mathrm{OD}_{600}=0.6-0.8\right)$, the culture was centrifuged at $3.3 \times \mathrm{g}$ for $10 \mathrm{~min}$. Bacteria were once more suspended in LB medium containing ampicillin $(100 \mu \mathrm{g} / \mathrm{mL})$ and incubated in a $0.1 \mathrm{mM}$ IPTG overnight at $30^{\circ} \mathrm{C}$. Bacteria were harvested and resuspended in $50 \mathrm{mM} \mathrm{NaH}_{2} \mathrm{PO}_{4}, \mathrm{pH}$ $7.5,300 \mathrm{mM} \mathrm{NaCl}, 10 \mathrm{mM}$ imidazole (1/10 of the culture volume). The bacteria were then sonicated for $30 \mathrm{~min}$. The cell lysate was centrifuged at $17.5 \times \mathrm{g}$ for $15 \mathrm{~min}$. The supernatant containing the scFvs were concentrated on $1 \mathrm{~mL}$ of $50 \%$ slurry Ni-NTA agarose (Qiagen, Valencia, CA) at $4^{\circ} \mathrm{C}$ by shaking for at least two hours. After washing with $50 \mathrm{mM}$ $\mathrm{NaH}_{2} \mathrm{PO}_{4}, \mathrm{pH} 7.5,300 \mathrm{mM} \mathrm{NaCl}, 10 \mathrm{mM}$ imidazole, bound scFvs were eluted by $50 \mathrm{mM} \mathrm{NAH}_{2} \mathrm{PO}_{4} \mathrm{pH} 7.5,300 \mathrm{mM}$ $\mathrm{NaCl}, 250 \mathrm{mM}$ imidazole.

Recombinant GST fusion RhoA protein was expressed and purified from a protease deficient strain (E. coli BL21).

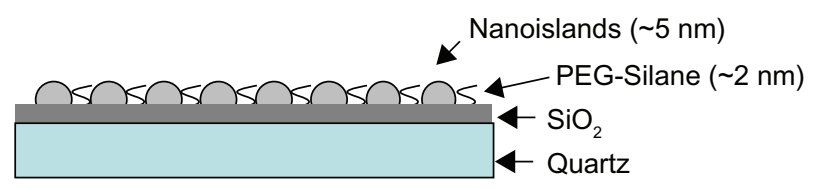

Figure 4 Schematic representation of a quartz functionalized with an antibiofouling PEG-silane coating plus $5 \mathrm{~nm}$ size Ni nanoislands.
Bacteria were grown at $37^{\circ} \mathrm{C}$ in 1 liter of $\mathrm{LB}$ medium containing ampicillin $(100 \mu \mathrm{g} / \mathrm{mL})$ until an $\mathrm{OD}_{600}=0.6-0.8$ was reached. Protein expression was induced for overnight incubation in $0.1 \mathrm{mM}$ isopropyl- $\beta$-D-glactopyranoside (IPTG) at $20^{\circ} \mathrm{C}$, and cells were harvested. Bacteria pellets were frozen at $-20^{\circ} \mathrm{C}$ for $15-20$ minutes, and subsequently resuspended at $4^{\circ} \mathrm{C}$ in $50 \mathrm{mM}$ Tris- $\mathrm{HCl}, \mathrm{pH} 7.5,150 \mathrm{mM}$ $\mathrm{NaCl}, 5 \mathrm{mM} \mathrm{MgCl}$, and triton $\times 1000.1 \%$ at $1: 10$ of the original culture volume. Cells were incubated in ice for 30 minutes then sonicated for five rounds of 15 seconds. Lysate was centrifuged at $12.500 \times \mathrm{g}$ for $30 \mathrm{~min}$. at $4^{\circ} \mathrm{C}$. The GST-RhoA containing supernatant was purified using the Magnet GSTTM protein purification system (Promega, Madison, WI) according to the manufacturer's instructions.

All purified recombinant proteins were applied to PD-10 desalting columns (GE Healthcare, Piscataway, NJ) equilibrated with HEPES $10 \mathrm{mM}$ pH 7.5, $150 \mathrm{mM} \mathrm{NaCl}$, $5 \mathrm{mM} \mathrm{MgCl}_{2}$ to remove glutathione and imidazole.

Samples were analyzed using SDS-PAGE and Coomassie blue staining and then stored at $4^{\circ} \mathrm{C}$ for short term storage or $-70^{\circ} \mathrm{C}$ with $15 \%$ glycerin for long term storage. Finally, for the Rho GTP loading, recombinant GST-RhoA purified using glutathione beads, was loaded with $0.1 \mathrm{mM}$ of GDP or GTPgS in Hepes $10 \mathrm{mM}, 150 \mathrm{mM} \mathrm{NaCl}, 5 \mathrm{mM} \mathrm{MgCl}$ at $37^{\circ} \mathrm{C}$ for 1 hour.

\section{ELISA tests}

HisGrab $^{\text {TM }}$ nickel coated 96-well plates (Pierce, Rockford, IL) were incubated for 1 hour at RT with $100 \mu \mathrm{L}$ of purified scFv F7N1N2 $(6 \mu \mathrm{g} / \mathrm{mL})$ in phosphate-buffered saline (PBS). The wells were washed three times with PBS Tween $0.05 \%$, and $100 \mu 1$ of purified GST-RhoA loaded with GDP or GTPgS, was added at appropriate concentrations in PBS, $5 \mathrm{mM} \mathrm{MgCl}_{2}$. After 1 hour of incubation at RT, the wells were washed three times and incubated using anti-GST-HRP conjugate (GE Healthcare, RPN1236). The immunoreaction was developed using 3, 3', 5,5'-tetramethylbenzidine (34022; Pierce), stopped by adding $1 \mathrm{M}$ sulfuric acid, and results were measured using an ELISA reader (Multiskan Labsystems, Hopkinton, MA) at $\mathrm{OD}=450 \mathrm{~nm}$.

\section{Results and Discussion}

To validate the surface chemistry involved for preventing non-specific adsorption, we used $0.5 \mu \mathrm{M}$ of 6His-tagged green fluorescent proteins (GFP), onto different quartz surfaces (quartz-SiO 2 , PEG-silane treated quartz-SiO ${ }_{2}$, before and after nanoislands deposition). After incubation of the GFP, the surfaces were analyzed by fluorescence 
microscopy. Figure 5A corroborates that the PEG based coating acts as an efficient antibiofouling layer preventing the adsorption of proteins. Conversely, on the virgin $\mathrm{SiO}_{2}$ film (without any surface chemistry treatment), the adsorption is considerable (Figure 5B). It is also clear that the presence of $\mathrm{Ni}$ nanoislands changes the repartition of the GFP proteins on the surface compared to $\mathrm{SiO}_{2}$ surfaces (Figure 5C). It is not possible to obtain any molecular scale information by using simple fluorescence characterizations but the role of the different layers is clearly identified. The PEG-silane coating forms an efficient antibiofouling layer between the Ni nanoislands while the latter induce a robust linkage to the surface on specific sites.

All these observations were verified using QCM analysis and no noticeable trace of adsorption (no frequency shift) was observed in the case of a $\mathrm{SiO}_{2}$ quartz coated with PEG-silane, while reproducible frequency shifts were recorded with virgin $\mathrm{SiO}_{2}$ quartz and $\mathrm{SiO}_{2}$ quartz treated with $\mathrm{PEG}$-silane plus Ni nanoislands.

The first attempts to detect the specific interaction of RhoA proteins with probe molecules selective to their active form by QCM using quartz crystals treated with PEG and Ni nanoislands failed. During these experiments, we identified an unexpected adsorption of glycerin (glycerol) on both $\mathrm{SiO}_{2}$ surfaces and PEG coated surfaces. We thus concluded that minimizing the quantity of glycerine, normally used as a cryogenic protectant for proteins storage, was mandatory for our experiments. This so-called "glycerin-effect", not noticed in traditional ELISA tests, was found to mask completely the specific protein interaction we wanted to investigate by QCM. We thus determined the maximum glycerin percentage compatible with a convenient storage of the proteins and reliable QCM signals. As shown in Figure 6 , the incubation of a solution containing a ratio of $0.2 \%$ (in volume) of glycerin in HEPES buffer does not induce any significant frequency shift on quartz- $\mathrm{SiO}_{2}$ coated with PEG and generates only a small variation of $1 \mathrm{~Hz}$ on virgin $\mathrm{SiO}_{2}$ quartz. Conversely, higher glycerin levels

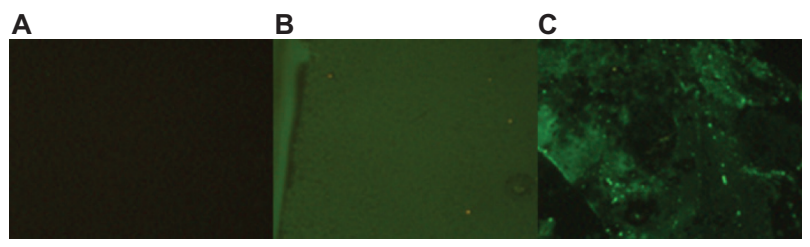

Figure 5 Fluorescence microscopy characterizations of the 6His-tagged green fluorescent proteins (GFP) adsorption, onto the different quartz surfaces. A) PEG-silane onto $\mathrm{SiO}_{2}$ B) $\mathrm{SiO}_{2}$ C) PEG-silane plus Ni nanoislands.
( $1 \%$ or $10 \%)$ were found to induce significant unspecific adsorption events on both types of surfaces.

When there was a percentage of glycerin higher than $0.2 \%$ mixed with the biomolecules of interest, the QCM signals exhibited a response comparable to those obtained for pure glycerine. To avoid this bias, the glycerin content in our solutions was kept below this level.

In the biomarker detection experiments we used Histagged antibody, single chain variable fragments ( $\mathrm{scFv}-$ F7N1N2) as a probe molecule, that bind selectively to the active antigen conformation RhoA, referred to RhoA-GTPgS with respect to the inactive antigen conformation of RhoA, then referred to RhoA-GDP. ${ }^{15}$ To verify the ability of this purified $\mathrm{scFv}$ to bind to nickel atoms while keeping its specificity for the active form of RhoA and before the final QCM tests, an ELISA test was conducted as follows: Ni-NTA microplates were coated with scFv F7N1N2. Specific interactions were
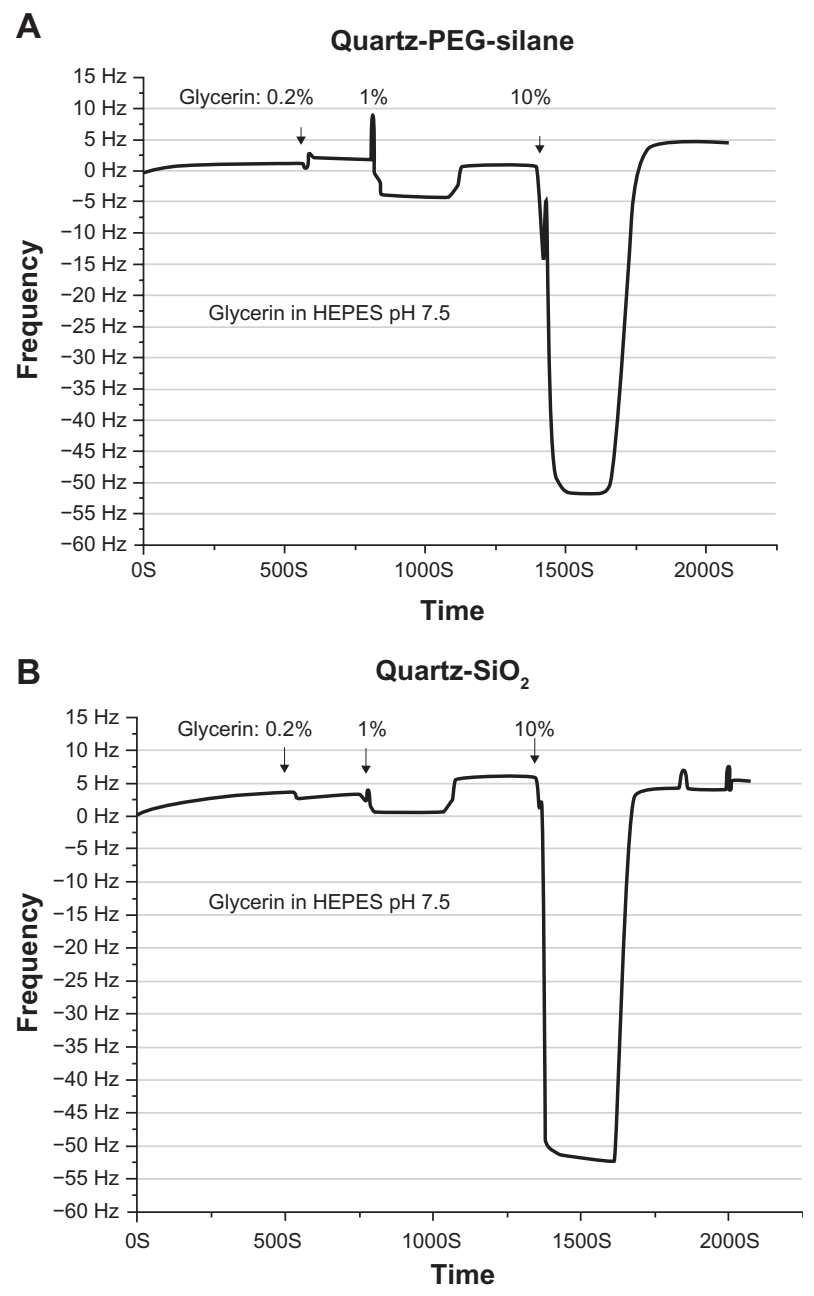

Figure 6 QCM signals obtained after the incubation of a solution of glycerin diluted in HEPES buffer at various concentrations (percentage in volume). A) quartz-SiO coated with PEG B) virgin quartz-SiO ${ }_{2}$. 


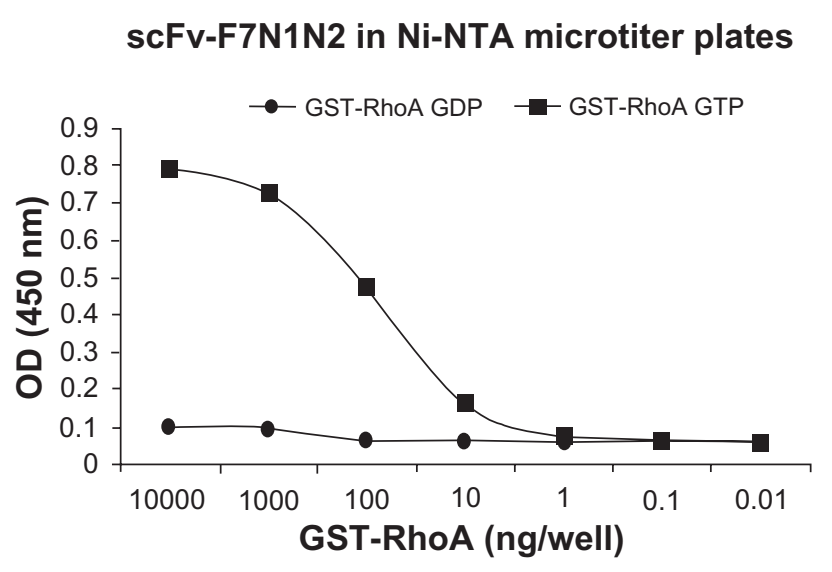

Figure 7 ELISA tests showing the selectivity of the selected probe molecule (His tagged scFv-F7NIN2) to the active conformation of RhoA proteins (RhoA GTPgS) compared to the inactive conformation (RhoA GDP) of the same protein.

measured by adding serial dilutions of GST-RhoA loaded with GDP or with GTPgS. The amount of GST-RhoA linked to the $\mathrm{scFv}$ was measured after adding an anti-GST-HRP conjugate. Figure 7 contains the results of these ELISA experiments, demonstrating the selectivity of our probe molecule to the active form of RhoA proteins and the good discrimination with respect to the inactive conformation of the same protein.

QCM experiments were finally performed at a flow rate of $60 \mathrm{~mL} / \mathrm{min}$ on quartz- $\mathrm{SiO}_{2}$ coated with PEG-silane and equipped with Ni nanoislands (similar to the scheme of Figure 4). Firstly, Hepes buffer $10 \mathrm{mM}, \mathrm{NaCl} 150 \mathrm{mM}$ plus $5 \mathrm{mM}$ of $\mathrm{MgCl}_{2}$ was injected into two similar independent quartz sensors, in a parallel manner. Quartz 1 was used to detect the active form of RhoA (RhoA GTPgS) and quartz 2 to detect the inactive form of RhoA (RhoA GDP). The results are depicted in Figure 8. At step 1, a $40 \mu \mathrm{g} / \mathrm{mL}$ $(730 \mathrm{nM})$ solution of scFv-F7N1N2 was injected into both fluidic channels. A frequency variation of around $70 \mathrm{~Hz}$ was observed in both quartz types, revealing the adsorption of the probe molecules on the surface covered with $\mathrm{Ni}$ islands. HEPES buffer was then injected for removing loosely bound molecules and for stabilizing the QCM signal. At step 2, a solution of RhoA GTPgS at $4 \mu \mathrm{g} / \mathrm{mL}(90 \mathrm{nM}$ ) was injected into quartz 1 and a solution of RhoA GDP at $4 \mu \mathrm{g} / \mathrm{mL}(90 \mathrm{nM})$ into quartz 2. As can be seen in Figure 8, the curve of the quartz 1 presents a frequency variation shift of around $5 \mathrm{~Hz}$ which can demonstrate a selective detection of the active form of RhoA (RhoA GTPgS), relating to the inactive form of RhoA (RhoA GDP) in which there is no noticeable frequency shift variation. However, we

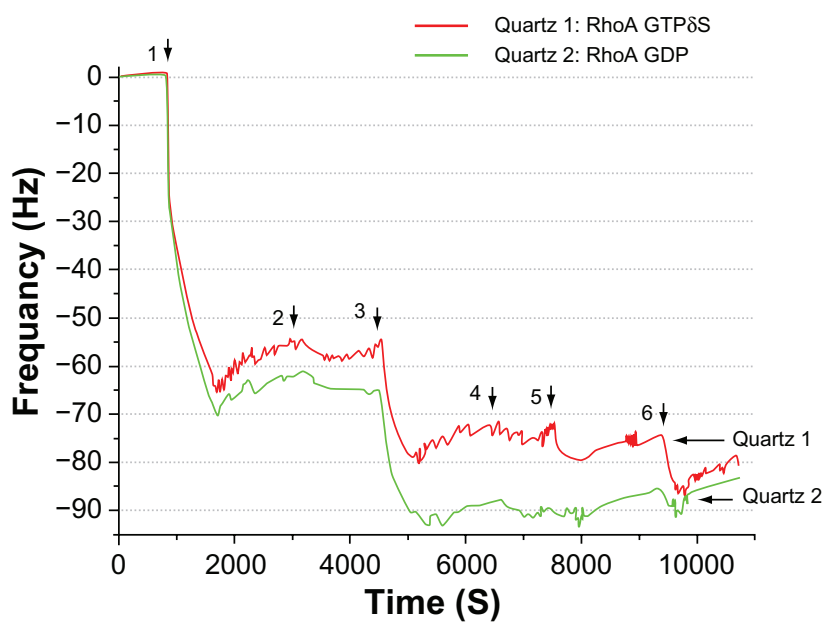

Figure 8 QCM Frequency variations of two identical quartz $\left(\mathrm{SiO}_{2} / \mathrm{PEG} / \mathrm{Ni}\right.$ nanoislands) subjected to different incubation steps.

wanted to be convinced of this selectivity so we decided to increase the surface density of probe molecules grafted on the Ni nanoislands by injecting and incubating, at step 3, a $200 \mu \mathrm{g} / \mathrm{mL}(3.6 \mu \mathrm{M})$ solution of (scFv)-F7N1N2 into both fluidic channels. Hence, an additional frequency shift of $\sim 25 \mathrm{~Hz}$ was observed in both reaction chambers (quartz 1 and quartz 2), which confirms that the surface was not initially saturated with probe molecules. At step 4, we injected the same concentration of RhoA proteins as at step 2, that is, $4 \mu \mathrm{g} / \mathrm{mL}(90 \mathrm{nM})$ of active and inactive antigens respectively, into quartz 1 and quartz 2 . A slight frequency shift was observed but could be distinctly attributed to a significant stable adsorption on the surface. We then increased the concentration of antigens up to $20 \mu \mathrm{g} / \mathrm{mL}$ (445 nM) (in step 5) and $40 \mu \mathrm{g} / \mathrm{mL}$ (900 nM) (in step 6) of active and inactive antigens respectively, into quartz 1 and quartz 2. In these cases, a clear adsorption signal was recorded in quartz 1 (7 Hz at step 5 and $10 \mathrm{~Hz}$ at step 6) while no trace of a clear adsorption could be noticed on quartz 2. The clear difference between the response of quartz 1 and quartz 2, indicates that the label-free detection of RhoA proteins in their active conformation can be achieved by QCM analysis. This result turned out to be possible only on quartz crystals equipped with nanometric size Ni nanoislands and was reproducibly obtained with the same batch of proteins.

\section{Conclusion}

We implemented a surface chemistry involving an antibiofouling coating of PEG-silane ( $<2 \mathrm{~nm}$ thick) onto a $\mathrm{SiO}_{2}$ thin film combined with a deposition of $5 \mathrm{~nm}$ size 
Ni islands. This specific surface chemistry performed onto $\mathrm{SiO}_{2}$ surfaces and the nanoscale roughness developed by the nanoislands turned out to be suitable for attaching probe molecules (antibody fragments) to the Ni islands through a Histidine tag, permitting label-free detection of the active conformation protein RhoA biomarkers and discriminating the inactive conformation. We found that glycerin, often used as a cryogenic protectant in molecular biology protocols, produced non-specific biomolecular interactions. A new protocol without glycerin has thus been developed and validated by ELISA technique. This work supports the viability of the concept of using Ni nanoislands as an anchoring surface layer enabling the detection of a specific conformation of a protein identified as a potential cancer biomarker.

Our aim was not to propose the QCM technology as a competitor to ELISA method, indeed the limit of detection, as shown in this paper, is poorer with QCM. Hence, QCM was used as a method for validating a specific surface chemistry on $\mathrm{SiO}_{2}$ surfaces that will be employed on a new type of label-free nanobiosensors based on MTJ-based nanodevices. This is expected to exhibit high sensitivity comparable to ELISA or nanowire-based FET sensors. ${ }^{25}$ These MTJ fabricated by silicon technology (e-beam lithography) use Ni nanoislands deposited between interdigitated nanoelectrodes on a silicon oxidized substrate, as the protocol validated here. We have demonstrated (results not yet published) that when a molecular complex is formed onto the Ni nanoislands, the room temperature electrical conductivity of these devices, dominated by so-called "coulomb blockade effect" change dramatically. This huge effect renders possible the label-free integrated and electrical detection of proteins in solution. The present publication was therefore a validation of the surface chemistry involved in these new kinds of devices. Hence, our results validate the adsorption of the probe molecules (conformationspecific antibody anti RhoA) on the Ni islands through His linkage, the efficiency of the antibiofouling layer around the islands, and the capability of our antibodies to detect specific conformational modifications of RhoA cancer biomarkers.

\section{Acknowledgments}

The authors thank Lionel Salmon from LCC-CNRS laboratory for helping with surface chemical treatments and Jérôme Chalmeau from LAAS-CNRS for help with AFM imaging. Also the TEAM service (Techniques et Equipements
Appliqués à la Microélectronique) for access to clean room facilities at LAAS-CNRS and ITAV (Institut des Technologies Avancées en science du Vivant) for the access to the bionanotechnology platform.

\section{Disclosure}

The authors report no conflicts of interest in this work.

\section{References}

1. Freitas RA. What is nanomedicine? Nanomedicine. 2004;1:101-109.

2. Kawasaki ES, Player A. Nanotechnology, nanomedicine, and the development of new, effective therapies for cancer. Nanomedicine. 2005;1:101-109.

3. Grodzinski P, Silver M, Molnar KL. Nanotechnology for cancer diagnostic: promises and challenges. Expert Rev Mol Diagn. 2006;6(3):307-318.

4. Palmer RE, Leung C. Immobilisation of proteins by atomic clusters on surfaces. Trends Biotech. 2006;25:48-55.

5. Tartaj P, Del Puerto Morales M, Veintemillas-Verdaguer S, González-Carreño T, Serna CJ. The preparation of magnetic nanoparticles for application in biomedicine. J Phys D Appl Phys. 2003;36: R182-R197.

6. Fonseca FC, Goya GF, Jardim RF, et al. Superparamagnetism and magnetic properties of Ni nanoparticles embedded in $\mathrm{SiO}_{2}$. Phys Rev B. 2002;B66:104406(1-5)

7. Likharev KK. Single electron devices and their applications. Proc IEEE. 1999;87(4):606-632.

8. Porath J, Carlsson JAN, Olsson, I, Belfrage G. Metal chelate affinity chromatography, a new approach to protein fractionation. Nature. 1975;258:598-599.

9. Schmitt J, Hess H, Stunnenberg HG. Affinity purification of histidinetagged proteins. Mol Biol Rep. 1993;18:223-230.

10. Rasooly A, Jacobson J. Development of biosensors for cancer clinical testing. Biosens Bioelectron. 2006;21:1851-1858.

11. Srinivas PR, Srivastava S, Hanash S, Wright GL. Proteomics in early detection of cancer. Clin Chem. 2001;47:1901-1911.

12. Etienne-Manneville S, Hall A. Rho GTpases in cell biology. Nature. 2002;420:629-635.

13. Sahai E, Marshall CJ. Rho GTpases and cancer. Nature Rev Cancer. 2002;2:133-142.

14. Mazieres J, Antonia T, Daste G, et al. Loss of RhoB expression in human lung cancer progression. Clin Cancer Res. 2004;10:2742-2750

15. Goffinet M, Chinestra P, Lajoie-Mazenc I, Medale-Giamarchi C, Favre G, Faye JC. Identification of a GTP-bound Rho specific scFv molecular sensor by phage display selection. BMC Biotech. 2008;8(34):1-14.

16. Richter R, Aydin D. Quartz crystal microbalance with dissipation monitoring (QCM-D). Practical Course in Biophysical Chemistry, Tiffin, OH: Heidelberg University; 2006.

17. Höök F, Rudh M. Quartz crystal microbalances (QCM) in biomolecular recognition. BTI Journal. 2005. Available from: http://www.biotech-online. com/uploads/tx_ttproducts/datasheet/quartz-crystal-microbalances(qcm)-in-biomacromolecular-recognition.pdf. Accessed Jan 10, 2010.

18. Backmann N, Zahnd C, Huber F. A label-free immunosensor array using single-chain antibody fragments. PNAS. 2005;102(41):14587-14592.

19. Johansson Alicia, Blagoi Gabriela, Boisen Anja. Polymer cantileverbased biosensor with integrated readout. Appl Phys Lett. 2006;89:173505173505-3

20. Ayela C, Nicu L. Micromachined piezoelectric membranes with high nominal quality factors in newtonian liquid media: A Lamb's model validation at the micro scale. Sens Actuators B Chem. 2007;123:860-868.

21. Malaquin L, Vieu C, Martinez C, Steck B, Carcenac F. Interdigitated nanoelectodes for nanoparticles. Nanotechnology. 2005;16:S240-S245. 
22. Martínez Rivas A, Séverac C, Carcenac F, Saya D, Nicu L, Vieu C. Wafer scale contact deposition of liquid solutions on interdigitated nanoelectrode devices for high sensitivity multiplexed biological analysis. Proceeding of the 34th International Conference on Micro and Nano Engineering (MNE) conference. 2008 Sep 15-18, Athens, Greece.

23. Kannan B, Castelino K, Chen FF, Majumdar A. Lithographic techniques and surface chemistries for the fabrication of PEG-passivated protein microarrays. Biosens Bioelec. 2006;21:1960-1967.
24. Papra A, Gadegaard N, Larsen N. Characterization of ultrathin poly(ethylene glycol) monolayers on silicon substrates. Langmuir. 2001;17:1457-1460.

25. Zheng G, Patolsky F, Cui Y, Cui Y, Wang UW, Lieber MC. Multiplexed electrical detection of cancer markers with nanowire sensor arrays. Nature Biotechnol. 2005;23(10):1294-1301.

\section{Publish your work in this journal}

The International Journal of Nanomedicine is an international, peerreviewed journal focusing on the application of nanotechnology in diagnostics, therapeutics, and drug delivery systems throughout the biomedical field. This journal is indexed on PubMed Central, MedLine, CAS, SciSearch $\AA$, Current Contents ${ }^{\circledR} /$ Clinical Medicine,
Journal Citation Reports/Science Edition, EMBase, Scopus and the Elsevier Bibliographic databases. The manuscript management system is completely online and includes a very quick and fair peer-review system, which is all easy to use. Visit http://www.dovepress.com/ testimonials.php to read real quotes from published authors. 